\title{
Molecular and immunological aspects of p53 and p53-autoantibodies in head and neck squamous cell carcinoma
}

\author{
C. CORDES, J. VON LINGEN, T. GÖRÖGH, P. AMBROSCH, S. GOTTSCHLICH and M. HOFFMANN
}

Department of Otorhinolaryngology, Head and Neck Surgery, Christian-Albrechts-University Kiel, Germany

Received May 22, 2009; Accepted August 11, 2009

DOI: $10.3892 /$ or_00000568

\begin{abstract}
The tumour suppressor protein p53 (wild-type $=$ wt-p53) is of major importance in the genetic integrity of the cell. Mutations of the p53-gene (mt-p53) are the most frequent genetic aberrations identified in different tumour entities. As analyzed in a wide variety of human malignomas, mtp53 evokes a specific immune response. Yet, the possible occurrence of p53-autoantibodies in patients with head and neck squamous cell carcinomas (HNSCC) correlated to p53mutations, p53 in sera and p53-overexpression in tissue has not been previously investigated. For the first time, the p53 status in 24 HNSCC patients was analyzed in the present study. The following parameters were investigated: analysis of mutation frequency of the p53-gene by direct sequencing of the exons 5-9, immunohistochemical detection of p53, measurement of the wt- and mt-p53-protein in sera by ELISA and p53-autoantibodies in sera by ELISA. Mutations of the p53-gene were detected in four $(17 \%)$ patients. Overexpression of wt-p53 was detected by immunohistochemistry in 18 out of $24(75 \%)$ tumours. In $8(33 \%)$ patients the p53-protein was also detectable in sera, whereas in just one of these eight patients p53-autoantibodies were detectable simultaneously. Overall 6 out of $24(25 \%)$ patients were found to be positive for serum p53-autoantibodies. Of these 6 cases, 5 could be assigned to tumours with immunohistochemically measurable wt-p53-overexpression. There was no correlation between p53-overexpression in tissue and p53-protein levels in sera or between p53-autoantibody levels in sera, nor in mutation frequency of the p53-gene and p53-overexpression in tissue. The results presented herein support the hypothesis that strong accumulation of p53 in the tissue is an important prerequisite for development of p53-autoantibodies. However, there must be further, yet unknown factors that
\end{abstract}

Correspondence to: Dr Christian Cordes, Department of Otorhinolaryngology, Head and Neck Surgery, Christian-Albrechts-University Kiel, Arnold-Heller-Strasse 14, D-24105 Kiel, Germany

E-mail: ccordes@hno.uni-kiel.de

Key words: p53, autoantibodies, cancer, squamous cell carcinoma influence the p53-autoantibody production because p53autoantibodies were not identified in sera in each case of p53-accumulation in the tissue.

\section{Introduction}

Every year 500,000 new cases of cancer in the head and neck region occur worldwide. More than $90 \%$ of these represent squamous cell carcinomas (HNSCC) (1). The main risk factors for developing HNSCC are the carcinogenic substances of tobacco smoke and alcohol. A correlation of elevated p53 expression in HNSCC with heavy smoking (2) and heavy smoking and drinking has been shown by Field et al (3). The p53-gene is the most frequently mutated gene in all human malignomas (4). More than $50 \%$ of human cancers show mutations of the p53-gene. p53-mutations in HNSCC are described in $40-60 \%$ of cases, thus they are one of the most frequent mutations in these tumours (5).

The p53-gene is located at the short arm of chromosome 17 band $17 \mathrm{p} 13$, consisting of 11 exons. Its product, the wildtype p53-protein (wt-p53), is a phosphoprotein localised in the cell nucleus. Wt-p53 contains a DNA-binding site, an oligomerization domain and a transcription activation domain. The molecular mass is $53 \mathrm{kD}$ and it consists of 393 amino acids (6).

In regular function, wt-p53 upregulates growth arrest and apoptosis-related genes in response to stress signals, thereby influencing programmed cell death, cell differentiation and cell cycle control mechanisms. wt-p53 yet can be chaperoned to the cytoplasm by the negative regulator MDM2, an E3 ubiquitin ligase. MDM2 is upregulated in the presence of active p53 and MDM2 polyubiquitinates p53 for proteasome targeting. p53 fluctuates between latent and active (DNAbinding) conformations and is differentially activated through post-translational modifications including phosphorylation and acetylation.

Especially mutations of exon 5-9 encoding the central binding site of the protein lead to severe misregulations. Mutations of this region are considered to cause malignant transformation of the cell. Moreover, the mutated p53-protein (mt-p53) inactivates wt-p53 by producing complex formation. It is implied that mutation of only one allele of p53 produces complete inactivation of p53 which is in contrast to other tumour suppressor genes (7). p53 alterations may result in release of the protein from the core of the cell into the serum 
Table I. Patient characteristics and overview of the results. ${ }^{\text {a }}$

\begin{tabular}{|c|c|c|c|c|c|c|c|c|c|}
\hline Patient & Gender & Age & Anatomical tumour site & TNM & Grading & Mutation & p53 AAB & p53 Protein & IHC \\
\hline 1 & Male & 52 & Base of tongue & T4N2cM0 & G3 & Exon 7 & & & +++ \\
\hline 2 & Male & 54 & Larynx & T3N0M0 & G2 & Exon 7 & & + & +++ \\
\hline 3 & Female & 43 & Larynx & T1N0M0 & $\mathrm{G} 2$ & & & + & - \\
\hline 4 & Male & 84 & Oropharynx & $\mathrm{T} 2 \mathrm{~N} 2 \mathrm{bM} 0$ & G3 & & & & +++ \\
\hline 5 & Male & 60 & Hypopharynx & T3N2bM0 & G2 & & & + & - \\
\hline 6 & Male & 42 & Hypopharynx & T4N3M1 & G3 & Exon 6 & & & ++ \\
\hline 7 & Male & 52 & Oropharynx & $\mathrm{T} 2 \mathrm{~N} 1 \mathrm{M} 0$ & $\mathrm{G} 2$ & & & & + \\
\hline 8 & Male & 60 & Larynx & T1N0M0 & $\mathrm{G} 2$ & & & + & +++ \\
\hline 9 & Male & 48 & Base of tongue & T1N0M0 & G2 & & & & ++ \\
\hline 10 & Male & 53 & Larynx & T4N2cM0 & G2 & & + & + & +++ \\
\hline 11 & Female & 59 & Oropharynx & $\mathrm{T} 2 \mathrm{~N} 1 \mathrm{M} 0$ & G3 & & & + & +++ \\
\hline 12 & Male & 75 & Hypopharynx & T4N2bM0 & G2 & & & & +++ \\
\hline 13 & Female & 39 & Oropharynx & T4N2cM0 & G3 & & + & & +++ \\
\hline 14 & Male & 46 & Hypopharynx & T3N2aM0 & G3 & Exon 5 & & & - \\
\hline 15 & Male & 62 & Hypopharynx & T4N3M1 & G1 & & & + & +++ \\
\hline 16 & Male & 49 & Larynx & T2N0M0 & G2 & & + & & +++ \\
\hline 17 & Male & 63 & Base of tongue & T2N1M0 & $\mathrm{G} 2$ & & + & & ++ \\
\hline 18 & Male & 59 & Base of tongue & T4N1M0 & G2 & & & & ++ \\
\hline 19 & Male & 70 & Larynx & T3N0M0 & G2 & & & & +++ \\
\hline 20 & Female & 60 & Oropharynx & T1N0M0 & G2 & & & & - \\
\hline 21 & Male & 64 & Hypopharynx & T3N2bM0 & $\mathrm{G} 2$ & & & & - \\
\hline 22 & Male & 46 & Base of tongue & T1N2bM0 & G3 & & & + & +++ \\
\hline 23 & Male & 62 & Oropharynx & T4N1M0 & G2 & & + & & - \\
\hline 24 & Male & 45 & Larynx & $\mathrm{T} 2 \mathrm{~N} 1 \mathrm{M} 0$ & G2 & & + & & +++ \\
\hline
\end{tabular}

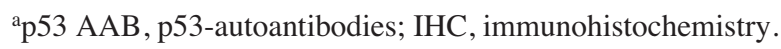

and this may lead to p53-autoantibody production. Crawford and colleague (8) first described this phenomenon in breast carcinoma which is reported for all cancers in $5-40 \%$ of the cases.

The role and the clinical impact of p53-autoantibodies are discussed controversially. The sensitivity of p53-autoantibodies as tumour marker in cancer patients is low, whereas the specificity as a tumour marker is high, since these autoantibodies only appear in cancer patients and in chronic inflammatory disease (9). As established for breast and bronchial carcinomas, the occurrence of p53-autoantibodies seems to be associated with poor prognosis of the patients (10). Furthermore, for patients with bladder and colon cancer serum anti-p53 antibody positive tumours had a worse prognosis than those with negative serum levels, regardless of the p53 status of the tumour (11). For HNSCC, the correlation of autoantibody occurrence and poor prognosis seems to be true as well, yet without statistical significance (12). The induction and formation process of p53-autoantibodies is poorly understood. It is hypothesized that p53overexpression in the tissue might be capable of activating the production of p53-autoantibodies in sera (13). Other reasons for $\mathrm{p} 53$-autoantibody production might be structural alterations of p53-protein due to mutation of the protein. The p53-genemutations may lead to a modified protein formation and newly expressed epitopes of the p53-protein subsequently leading to modification of the central binding site of the protein and to develop antigenic character (14). However, Schlichtholz et al (15) and Maass et al (16) suggest that tumour cell necrosis might release p53 from the cell subsequently accumulating in the serum thus being presented to the immune system. If this was true, not only p53-autoantibodies but also p53 itself should be detectable in sera.

To contribute to this issue, we examined p53 genetic mutation, p53 protein-expression in tissue, wt-p53 and mt-p53 expression in sera, and p53-autoantibodies in sera. As to our knowledge, this is the first study comparing the results of these parameters.

\section{Materials and methods}

Patients. Serum and tissue specimens were obtained from 24 patients with HNSCC. Five tumours were localised in the base of tongue, 6 in the oropharynx, 6 in the pharynx and 7 in the larynx. All patients were treated in the Department of Otorhinolaryngology, Head and Neck Surgery, ChristianAlbrechts-University Kiel, Germany. The age of the patients was from 39 to 84 years $(56.1 \pm 10.9)$. Of the 24 patients, 20 were male and 4 female. Tumours were classified according to the TNM classification of the Union Internationale Contre 

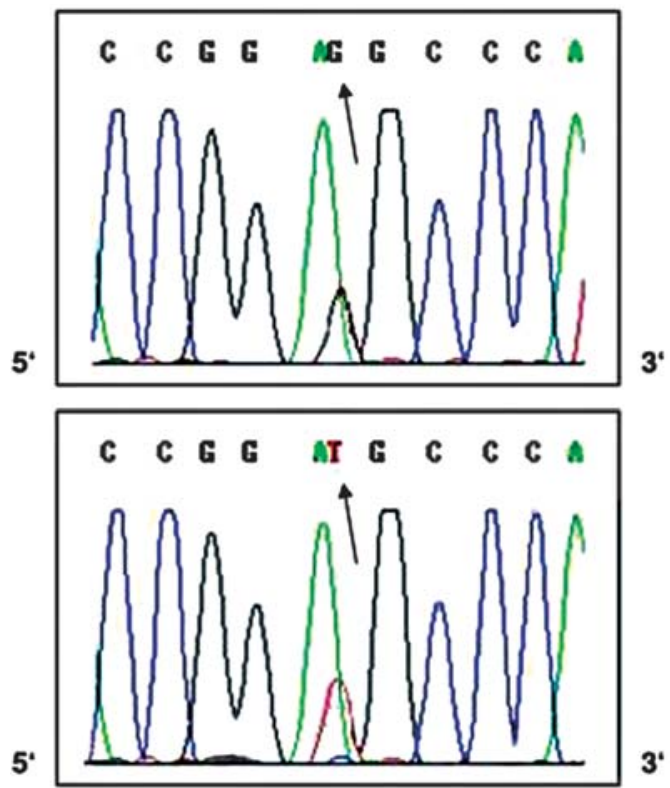

Figure 1. Part of a 5'-3' sequence profile of exon 7. Arrows indicate heterocygotic point mutation in codon 249 . The upper image shows the wild-type signal $(\mathrm{G})$, the lower marks the mutated gene locus $(\mathrm{T})$.

Cancer (UICC) from 1987 and by grade of differentiation, age and gender (Table I). Pretherapeutic serum probes were obtained and stored in liquid nitrogen. All samples were retrieved following informed consent approved by the local ethics committee (AZ: D413/03).

Sequencing of exons 5-9. Prior to sequencing, DNA was extracted using the DNAeasy kit (Qiagen, Germany) and amplified by PCR using oligonucleotides specific for exon 5-9. PCR was carried out using a thermocycler with following conditions for $100 \mu 1$ reaction mixture: $10 \mu 1$ 10-fold PCR buffer, $2.5 \mu \mathrm{l}$ of $50 \mathrm{mM} \mathrm{MgCl} 2,0.5 \mu \mathrm{l}$ of $5 \mathrm{U} / \mu 1 \mathrm{Taq}$ DNA polymerase, $2 \mu \mathrm{l}$ of $10 \mathrm{mM}$ dNTP, $1 \mu \mathrm{l}$ of $100 \mu \mathrm{M}$ sense-antisense primer each and $100 \mathrm{ng}$ template DNA in $83.1 \mu 1 \mathrm{H}_{2} \mathrm{O}$ (all PCR reagents from Boehringer, Germany). PCR was run for 35 cycles at $96^{\circ} \mathrm{C}$ for $10 \mathrm{sec}$ denaturizing, $59^{\circ} \mathrm{C}$ for $5 \mathrm{sec}$ annealing and $60^{\circ} \mathrm{C}$ for 4 min elongation.

After PCR the amplicons were purified using a GFX purification kit (Pharmacia, Germany), then upstream and downstream sequenced according to the method previously described by Sanger et al (17) with a computer assisted genetic analyzer (ABI Prism 310 genetic analyzer, Applied Bio-systems, Germany). The resulting sequence data was compared with p53 wild-type sequences (Fig. 1).

Immunohistochemistry. Routine staining of each tissue probe was done by haematoxylin and eosin (H\&E). Immunohistochemical staining was performed using a primary monoclonal antibody (DO-7) against the protein p53 (Dako, Denmark). Negative controls were obtained by omission of the primary antibody and by incubating the primary antibody with specific blocking peptide in 5- to 10-fold molar excess before staining. Then immune histochemical core staining was evaluated from 0 to 3 fold positive (Fig. 2).

Quantitative measurement of p53. Sandwich-ELISA kit (Oncogene Research Products, USA) was used to measure p53-protein and p53-autoantibodies in the sera. Photometric measurement was carried out at 490 and $630 \mathrm{~nm}$, respectively, using the ELISA-Reader MR500 (Dynatech, Germany). Samples with an average p53-protein concentration of $\geq 300 \mathrm{pg}$ / $\mathrm{ml}$ were assessed as positive.

Statistical analysis. Fischer's exact test with SPSS statistical software (version 13.0) was used to assess significant differences. The data were considered statistically significant at $\mathrm{p} \leq 0.05$.

\section{Results}

Of the 24 tumour samples used, p53-gene sequencing revealed mutations in 4 out of 24 cases (17\%). In all cases missense mutations occurred i.e. mutations in which nucleotides were changed, resulting in a codon encoding for a different amino acid (Table II).

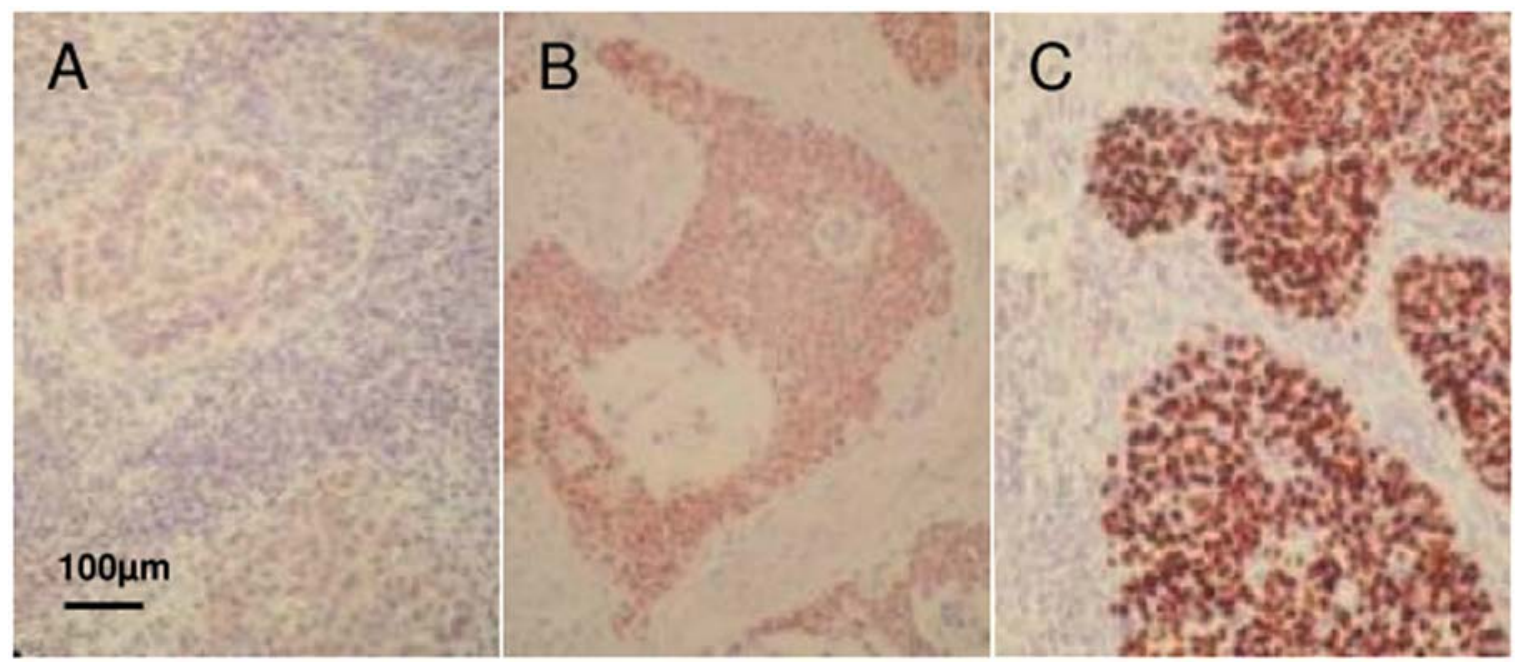

Figure 2. Immunohistochemical staining showing weak (A), moderate (B), and strong (C) nuclear accumulation of p53 (brown) in invasive growing squamous cell carcinomas. 
Table II. Gene locus, change in nucleotide sequence and resulting amino acid change in the detected mutations of p53 gene.

\begin{tabular}{lccc}
\hline Mutated regions & Codon & Change in nucleotide sequence & Amino acid change \\
\hline Exon 5 & 173 & Transversion GTG $\rightarrow$ TTG & Valine $\rightarrow$ leucine \\
Exon 6 & 214 & Transversion CAT $\rightarrow$ GAT & Histidine $\rightarrow$ aspartic acid \\
Exon 7 & 242 & Transversion TGC $\rightarrow$ GGC & Cysteine $\rightarrow$ glycine \\
Exon 7 & 249 & Transversion AGG $\rightarrow$ ATG & Arginine $\rightarrow$ methionine \\
\hline
\end{tabular}

H\&E staining confirmed the presence of HNSCC in the tissue samples which were evaluated semi-quantitative by means of immunohistochemistry. p53-overexpression was featured in 18 out of 24 cases $(75 \%)$. Weak expression was detected in only 1 case, moderate expression in 4 cases and strong p53-expression in 13 cases. In the remaining 6 cases no $\mathrm{p} 53$-expression was detectable.

Wt-p53 and mt-p53 protein was quantified by ELISA in serum samples taken from 24 tumour patients. Overall, p53 concentrations were between 66 and $1040 \mathrm{pg} / \mathrm{ml}$. Eight out of the 24 serum samples $(33 \%)$ contained $>300 \mathrm{pg} / \mathrm{ml}$ and were considered as positive for $\mathrm{p} 53$ in sera.

Considering the total number of samples analysed, there was no significant correlation between p53-autoantibodies in sera and $\mathrm{p} 53$-overexpression in the tissue $(\mathrm{p}=0.5)$. p53autoantibodies were detected in sera of only 6 out of 24 patients (25\%). However, in 5 out of these 6 patients a significant correlation between $\mathrm{p} 53$-autoantibody production in sera and $\mathrm{p} 53$-overexpression in the tissue could be found $(\mathrm{p}=0.03)$, suggesting that $\mathrm{p} 53$-overexpression is a necessary precondition for $\mathrm{p} 53$-autoantibody production. It remains unknown why so many patients with $\mathrm{p} 53$-overexpression do not produce $\mathrm{p} 53$ autoantibodies in the sera.

Comparing the clinical and histopathological parameters (TNM, grading, anatomical tumour site, gender and age) with the rate of p53-mutations, p53-overexpression, p53 in sera and p53-autoantibody production showed no statistically significant correlation.

\section{Discussion}

Mutations of p53 in HNSCC are described in 40-60\% of cases, thus they are one of the most frequent mutations in these tumours (5). The results presented herein, show a p53mutation rate of $17 \%$ which is rather low compared to other studies of SCC in the head and neck region. The low incidence of mutations presented herein might be due to the applied detection method for mutations. Direct sequencing of the exons 5-9 was performed following amplification by PCR, representing a technique which is known to be very sensitive. Yet, the method is risky to overlook mutations in case wt-p53 sequences of contaminating regular cells disturb the signal of the mutated cells. In studies reporting higher mutation rates single-strand conformation polymorphism (SSCP) was applied, a technique which has shown rather frequent false positive results (1).

In 8 out of 24 patients (33\%) investigated, p53 itself was detectable in serum. Regularly p53 can not be detected in patients sera since p53 is located in the nucleus with a short biological half-life (6-20 min). Presumably, p53-accumulation occurs in tumour cells and consecutively p53 is released into sera due to tumour cell necrosis. The described process of protein release might be the prerequisite for the immunological response and the development of p53-autoantibodies. However, in this study there was no significant correlation between p53-accumulation in the tissue and the occurrence of p53-autoantibodies.

The first immunological response against p53 was described in 1979 (18). It was discovered rather by accident while researching the popular tumour virus SV40. p53 is a complex-builder with the $\mathrm{T}$ antigen of the virus. p53autoantibodies were first shown by Crawford et al in 1982 (8). Later on, measurable p53-autoantibody levels in sera were demonstrated in various malignancies (10-14).

Depending on tumour type and method of analysis the rate of p53-autoantibodies varied between 5 and $40 \%(19,20)$. In the present study, $25 \%$ of patients autoantibodies against p53 could be shown by ELISA in pre-therapeutical serum. This quantity is consistent with previously studies on p53 autoantibodies in HNSCCs $(12,16,21)$. Rates higher than $\sim 40 \%$ are rarely described (22).

Two dominating hypothesis for the development of p53autoantibodies are subject of controversy in literature. Lubin and colleagues (13) hypothesized that mutations of the p53gene produce a modified protein formation and thus newly expressed epitopes of the p53-protein subsequently develop antigenic character. Winter et al (14) detected p53-autoantibodies only in patients with a point mutation in exon 7 , whereas no mutation in exon 8 was detected. The significant correlation between mutation and p53-autoantibodies has been supported by other studies $(23,24)$. Yet, there are studies $(25,26)$ showing no significant correlation between p53mutation and p53-autoantibody development. One of several reasons arguing against p53-mutation as single cause for autoantibody production is that $\mathrm{p} 53$-autoantibodies bind to epitopes that are expressed in both, mt-p53 and wt-p53 (27). Moreover, these epitopes are rather located on the $\mathrm{C}$ - and $\mathrm{N}$-terminus of the protein, while mutations mainly occur in the central binding site of the protein (28).

No correlation between p53-mutation and occurrence of p53-autoantibodies was observed. In four out of 24 cases p53-mutations were detected. However, none of these cases showed serological evidence of p53-autoantibodies. Thus, it can be concluded that mutations of the p53-gene leading to a modified protein formation and newly expressed epitopes of the p53-protein which subsequently develop antigenic characteristics cannot be supported by the data presented herein. Furthermore, there was no correlation between 
occurrence of p53 in the serum and to TNM stage, grading, and tumour site.

Another hypothesis favoured by different authors $(16,29,30)$ implies an overexpression of p53 in the tissue with subsequent production of p53-autoantibodies. p53 accumulation in the tissue might be a result of tumour cell necrosis with release of p53 out of its natural compartment (15). An internalisation of antigen presenting cells might finally give a B-cell reaction (16).

In concordance with this hypothesis the results presented herein demonstrate a $75 \%$ p53-overexpression in tumour tissue. This result is at the upper limit of previously described results with a p53-overexpression rate between $35 \%$ (31) and $84 \%$ (32). Altogether, the results of this analysis do not show a significant correlation between p53-mutation and p53overexpression in the tissue. However, in almost all cases of circulating p53-autoantibodies also an overexpression of p53 in the tissue could be seen, indicating that p53-overexpression in the tissue is a necessary precondition for p53-autoantibody production in the tested cases.

In conclusion, the results presented herein support the hypothesis that strong accumulation of p53 in the tissue is an important prerequisite for development of p53-autoantibodies. However, there must be further, still unknown factors that influence the p53-autoantibody production because p53autoantibodies were not identified in sera in each case of p53-accumulation in the tissue. Neither the initial proposition of $\mathrm{p} 53$-overexpression in the tissue with consecutive p53 release into the tissue and subsequent production of p53autoantibodies nor the assumption that mutations in the p53 genome lead to the production of p53-autoantibodies can be confirmed by the data demonstrated in this study.

\section{References}

1. Koh JY, Cho NP, Kong G, Lee JD and Yoon K: p53 mutations and human papillomavirus DNA in oral squamous cell carcinoma: Correlation with apoptosis. Br J Cancer 78: 354-359, 1998

2. Field JK, Spandidos DA, Malliri A, Gosney JR, Yiagnisis M and Stell PM: Elevated p53 expression correlates with a history of heavy smoking in squamous cell carcinoma of the head and neck. Br J Cancer 64: 573-577, 1991

3. Field JK, Spandidos DA and Stell PM: Overexpression of p53 gene in head-and-neck cancer, linked with heavy smoking and drinking. Lancet 339: 502-503, 1992.

4. Levine AJ, Momand J and Finlay CA: The p53 tumour suppressor gene. Nature 351: 453-456, 1991.

5. Woods DB and Vousden KH: Regulation of p53 function. Exp Cell Res 264: 56-66, 2001.

6. Miller C, Mohandas T, Wolf D, Prokocimer M, Rotter V and Koeffler HP: Human p53 gene localized to short arm of chromosome 17. Nature 319: 783-784, 1986.

7. Strickler JG, Zheng J, Shu Q, Burgart LJ, Alberts SR and Shibata D: p53 mutations and microsatellite instability in sporadic gastric cancer. Cancer Res 54: 4750-4755, 1994.

8. Crawford LV, Pim DC and Bulbrooke AD: Detection of antibodies against the cellular protein p53 in sera from patients with breast cancer. Int J Cancer 30: 403-408, 1982.

9. El Sayed ZA, Farag DH and Eissa S: Tumor suppressor protein p53 and anti-p53 autoantibodies in pediatric rheumatological diseases. Pediatr Allergy Immunol 14: 229-233, 2003.

10. Peyrat JP, Bonneterre J, Lubin R, Vanlemmens L, Fournier J and Soussi T: Prognostic significance of circulating p53 antibodies in patients undergoing surgery for locoregional breast cancer. Lancet 345: 621-622, 1995.

11. Gumus E, Erdamar S, Demirel G, Horasanli K, Kendirci M and Miroglu C: Association of positive serum anti-p53 antibodies with poor prognosis in bladder cancer patients. Int J Urol 11: 1070-1077, 2004.
12. Gottschlich S, Hoffmann M, Maass JD, et al: p53 autoantibodies as tumor marker in head and neck squamous cell cancer. Anticancer Res 23: 913-915, 2003.

13. Lubin R, Schlichtholz B, Bengoufa D, et al: Analysis of p53 antibodies in patients with various cancers define B-cell epitopes of human p53: distribution on primary structure and exposure on protein surface. Cancer Res 53: 5872-5875, 1993.

14. Winter SF, Minna JD, Johnson BE, Takahashi T, Gazdar AF and Carbone DP: Development of antibodies against p53 in lung cancer patients appear to be dependent on the type of p53 mutation. Cancer Res 52: 4168-4174, 1992.

15. Schlichtholz B, Legros Y, Gillet D, et al: The immune response to p53 in breast cancer patients is directed against immunodominant epitopes unrelated to the mutational hot spot. Cancer Res 52: 6380-6384, 1992.

16. Maass JD, Gottschlich S, Goeroegh T, Lippert BM and Werner JA: Head and neck cancer and p53-immunogenicity. Anticancer Res 17: 2873-2874, 1997.

17. Sanger F, Nicklen S and Coulson AR: DNA sequencing with chain-terminating inhibitors. Proc Natl Acad Sci USA 74: 5463$5467,1977$.

18. Lane DP and Crawford LV: T antigen is bound to a host protein in SV40-transformed cells. Nature 278: 261-263, 1979.

19. Hammel P, Boissier B, Chaumette MT, et al: Detection and monitoring of serum p53 antibodies in patients with colorectal cancer. Gut 40: 356-361, 1997.

20. Sidransky D, Tokino T, Hamilton SR, et al: Identification of ras oncogene mutations in the stool of patients with respectable colorectal cancer. Science 256: 102-105, 1992.

21. Gottschlich S, Folz BJ, Goeroegh T, Lippert BM, Maass JD and Werner JA: A new prognostic indicator for head and neck cancer-p53 serum antibodies? Anticancer Res 19: 2703-2705, 1999.

22. Wollenberg B, Jan NV, Pitzke P, Reiter W and Stieber P: Antip53 antibodies in serum of smokers and head and neck cancer patients. Anticancer Res 17: 413-418, 1997.

23. Ralhan R, Arora S, Chattopadhyay TK, Shukla NK and Mathur M: Circulating p53 antibodies, p53 gene mutational profile and product accumulation in esophageal squamous-cell carcinoma in India. Int J Cancer 85: 791-795, 2000.

24. von Brevern MC, Hollstein MC, Cawley HM, et al: Circulating anti-p53 antibodies in esophageal cancer patients are found predominantly in individuals with p53 core domain mutations in their tumors. Cancer Res 56: 4917-4921, 1996.

25. Angelopoulou K, Yu H, Bharaj B, Giai M and Diamandis EP: p53 gene mutation, tumor p53 protein overexpression, and serum p53 autoantibody generation in patients with breast cancer. Clin Biochem 33: 53-62, 2000.

26. Collet B, Raoul JL, Le Berre N, et al: Serum anti-p53 antibodies in patients with squamous cell carcinoma of the esophagus: Comparison with p53 alterations and lymph node invasion. Int J Oncol 11: 617-621, 1997.

27. Labrecque S, Naor N, Thomson D and Matlashewski G: Analysis of the anti-p53 antibody response in cancer patients. Cancer Res 53: 3468-3471, 1993.

28. Lubin R, Schlichtholz B, Teillaud JL, et al: p53 antibodies in patients with various types of cancer: assay, identification and characterization. Clin Cancer Res 1: 1463-1469, 1995.

29. Lavieille JP, Lubin R, Soussi T, Reyt E, Brambilla C and Riva C: Analysis of p53 antibody response in patients with squamous cell carcinoma of the head and neck. Anticancer Res 16: 2385-2388, 1996.

30. Davidoff AM, Iglehart JD and Marks JR: Immune response to p53 is dependent upon p53/HSP70 complexes in breast cancers. Proc Nat Acad Sci USA 89: 3439-3442, 1992.

31. Warnakulasuriya S, Soussi T, Maher R, Johnson N and Tavassoli M: Expression of p53 in oral squamous cell carcinoma is associated with the presence of $\operatorname{IgG}$ and $\operatorname{IgA}$ p 53 autoantibodies in sera and saliva of the patients. J Pathol 192: 52-57, 2000.

32. Dolcetti R, Doglioni C, Maestro R, et al: p53 over-expression is an early event in the development of human squamous-cell carcinoma of the larynx: genetic and prognostic implications. Int J Cancer 52: 178-182, 1992. 